\title{
Clinical and Economic Impact of Antimicrobial Stewardship Interventions Reported Among Hospital Inpatients in The Middle East: a Systematic Review Protocol
}

Binny Thomas ( $\sim$ binnyinhmc@gmail.com )

Robert Gordon University https://orcid.org/0000-0003-1065-8343

Pallivalappila Abdulrouf

Hamad Medical Corporation

Wessam Elkassem

Hamad Medical Corporation

Fatema Al Hail

Hamad Medical Corporation

Zachariah Nazar

Qatar University

Ziad Nasr

Qatar University

Rajvir Singh

Hamad Medical Corporation

Hussam Al Soub

Hamad Medical Corporation

Derek Stewart

Qatar University

Moza Al Hail

Hamad Medical Corporation

Protocol

Keywords: AMR, patient, public health, Economic, Antimicrobial

Posted Date: August 2nd, 2020

DOI: https://doi.org/10.21203/rs.3.rs-50679/v1

License: (c) (1) This work is licensed under a Creative Commons Attribution 4.0 International License.

Read Full License 
Page $2 / 19$ 


\section{Abstract}

Antimicrobial Resistance (AMR) is a major global concern, negatively impacting public health outcomes (1-5), patient safety $(3,6-8)$ and poses enormous burden on economic development and security (8-16). Internationally, data suggests, at any given time, approximately one-third of the hospitalized patients and over two-thirds of critically ill patients are prescribed antimicrobials and more than half of these prescribed antimicrobials are deemed inappropriate $(5,17,18)$.

\section{Expertise}

Our research team covers key areas of expertise required for this review, includes, clinical pharmacy specialists, infectious disease experts, health policy researchers etc. We have worked together previously with a proven track record of researching fields of obstetric and perinatal population. We have also managed complex studies within time and budget limitations.

\section{Background}

\section{Global overview and clinical impact of antimicrobial resistance}

Antimicrobial Resistance (AMR) is a major global concern, negatively impacting public health outcomes (1-5), patient safety $(3,6-8)$ and poses enormous burden on economic development and security (8-16). Internationally, data suggests, at any given time, approximately one-third of the hospitalized patients and over two-thirds of critically ill patients are prescribed antimicrobials and more than half of these prescribed antimicrobials are deemed inappropriate $(5,17,18)$.

The health impact of AMR is catastrophic, presenting significant challenges to treating microbial infections (19-21). Various factors contributing to AMR include misuse of antimicrobials, emergence of resistant bacterial genes, dearth of newer antimicrobial agents, and lack of coordinated antimicrobial stewardship programs (ASPs) $(2,8,9,22)$. A report from the Centers for Disease Control and Prevention (CDC) in the US, estimates at least 2.8 million patients with serious infections are resistant to at least one or more antibiotics that were actually intended to treat those infections, and at least 44000 of these patients die yearly as a result of the multidrug resistant infections (23). Data from Europe reveals 23000 deaths occur annually due to antibacterial resistance (24). AMR data from the region reported, $20 \%$ mortality among pediatric hematological patients (25), 40\% from a tertiary hospital (26) and 60 to $90 \%$ of the ventilator associated pneumonia were found resistant to most of the existing antibiotics (27).

\section{Economic impact of antimicrobial resistance}

In addition to destabilizing healthcare goals, AMR is associated with substantive economic consequences. An estimated US\$ 34 billion is spent annually on the treatment of antibiotic-resistant infections in US hospitals alone (28). Data from the World Bank estimates an increase in healthcare cost of up to one trillion \$US annually and an additional 10 million people worldwide will die every year due to 
AMR by 2050 (11). The Centre for Disease Prevention and Control in European Union estimates an annual healthcare cost of $€ 1.5$ billion due to AMR in the form of lost productivity, wages, extended course of therapy and additional medical expenses etc. (29). An overall global scale of AMR is more difficult to quantify as economical and epidemiological data are scarce in many parts of the world (30), however available data indicate a considerable concern. By 2050, if no further interventions are made to combat AMR, the world economy would decline by anywhere between $0.06 \%$ and $3.1 \%(31,32)$.

\section{Importance of antimicrobial stewardship program}

In recognition to the magnitude of harm caused by AMR, the World Health Organization (WHO) and the United Nations at the General Assembly of 2016 recommended urgent implementation of country-level and institutional antimicrobial stewardship programs (ASPs) as a method of enhancing appropriate use of antimicrobials thus combating resistance (33). The Infectious Disease Society of America (IDSA) defined ASP as "a set of coordinated interventions designed to improve antimicrobial use in terms of selecting the appropriate agent, dose, route of administration, and therapy duration without compromising patient outcomes"(33).

The development of AMR is multifactorial and thus requires multifaceted, interventions to combat the spread of resistance in healthcare settings $(2,7,23,30)$. ASP has evolved from a cost saving phenomenon to a tool dedicated to reducing the spread of microbial resistance by minimizing the misuse of antimicrobials. In the recent years, several studies have highlighted the positive impact of ASPs in terms of reducing resistance, antibiotic use and healthcare costs $(13,15,34,35)$.

Furthermore, in an effort to improve antimicrobial use in hospitals, the CDC recommends all acute care hospitals to implement ASPs. To support this initiative, the CDC released Core Elements of Hospital Antibiotic Stewardship Programs a framework specially designed to assist hospitals in both starting and expanding ASPs. The seven core elements are described in the table below, (36)

Davie et.al (5) classified antimicrobial stewardship interventions into (i) Persuasive - interventions that provide information for prescribers (such as audit, education, feedback etc.), (ii) Restrictive interventions aimed at setting rules or implementing standards (such as formulary restrictions, prescribing restrictions), (iii) Structural - providing support and quality control (decision support, electronic prescribing etc)

\section{Problem statement and evidence}

Several systematic reviews have evaluated the clinical and economic impact of ASPs $(5,13,34,35,37-40)$, however, limitations compromise their generalizability as they either focused on, critical care unit (39), specialized inpatient wards $(5,40)$, or outpatient units $(38)$. Whilst some reviews explicitly focused on pediatrics (20) and long-term residential care (41), few others reviewed the impact of antimicrobial stewardship interventions distinctly in western countries and Asia (34). Notably, most of the research on ASP and AMR has originated from North American and European healthcare centers. Middle East and the 
Persian Gulf has a dearth of epidemiological data on AMR, and the impact of antimicrobial stewardship practices remains largely unknown $(35,42)$.

The Middle East includes 15 strategically, politically and economically diverse countries: Bahrain, Egypt, Iraq, Iran, Jordan, the Kingdom of Saudi Arabia (KSA), Kuwait, Lebanon, Palestine, Qatar, Sultanate Oman, Syria, Turkey, the United Arab Emirates (UAE), and Yemen (43). Conversely, Middle East has its own share of antibiotic-resistance issues that stems from a wide range of problems, including selfprescribing $(44,45)$, misuse of antimicrobials $(35,45)$ over the counter sales $(46,47)$, poor infection control measures, poor understanding and unrealistic expectations among patients $(48,49)$, and most notably due to the recent population mobilization due to socio-economic developments, conflicts and multiple war crises (50). A recent report from the WHO has also highlighted that, due to poor economic status and lack of health care insurance, patients often approach pharmacists for their medications to avoid consultations costs (50). Moreover, the overall public awareness about the use of antimicrobials and its resistance in this region is reported to be poor $(48,49,51-55)$.

Recent systematic review related to ASP from Middle East, reported, strategies to assess appropriateness of antimicrobial prescribing and behaviors in selected Middle Eastern countries. However, no information about the quality of studies was provided and the review did not address an in depth clinical or economic impact of the stewardship program. Furthermore, albeit, all recent developments, the ASPs in the Middle East are suboptimal and still in their infancy (35). One further systematic review investigating the barriers and facilitators of implementing ASPs in Gulf Cooperation Council (GCC) stated that adoption of ASPs among hospitals and other healthcare settings remains low and largely under reported. Poor planning and lack of national strategies to combat AMR further hinders the implementation of ASPs in GCC (42). A PROSPERO registered systematic review protocol on aspects of CDC alignment of ASP implementation and its potential barriers and facilitators limited to GCC countries is in progress (56).

\section{Significance and aim of the study}

Considering the diverse healthcare systems, extensive geographic area, economy, ethnic background, cultural context and healthcare practices in the Middle East, there may be merit in conducting a systematic methodological review of published evidence to measure the efficacy of antimicrobial stewardship efforts in the region and to assess the subsequent clinical and economic outcomes. Given that, concerted efforts are being made by health authorities across the globe, particularly in this region, to combat AMR. However, hospital level data in terms of variation in stewardship practices, interventions used, antimicrobial consumption, and resistance across different countries in the Middle East has not been addressed till date.

The findings of this review are anticipated to provide scientific foundation to develop an evidence informed intervention to address the burdensome sequelae of antimicrobial resistance in the region. The findings will inform the policymakers about the importance of adoption of ASPs in hospitals. Rigorous and unbiased economic impact of ASPs will further inform decision makers and key stakeholders on how to allocate scarce healthcare resources in the most beneficial way. Considering the recent advancements 
in healthcare in the Middle East, an updated systematic review in relation to understanding the state of antimicrobial stewardship incorporating economic consequences in the Middle East is warranted. Furthermore, we anticipate that sharing the regional experience will facilitate other healthcare institutions to adopt ASPs.

\section{Research Question(s)}

The systematic review seeks to answer the following questions in relation to antimicrobial use in hospitals in Middle East,

1. What are the core elements of hospital antimicrobial stewardship tools/interventionsused/reported to improve antimicrobial use in hospitals?

2. What are the different outcome measuresreported?

3. What is the clinical and economic impactof antimicrobial stewardship interventions on antimicrobial prescribing and use in hospitals?

4. What are the improvement strategies reported/suggested that could reduce AMR within hospitals in Middle East?

\section{Methods}

The systematic review protocol was developed in accordance with the Preferred Reporting Items for Systematic Review and Meta-Analysis Protocols (PRISMA-P) guidelines (57) (Additional file 1). In order to reduce the variability and enhance the rigor and validity of results, the review protocol is registered in the PROSPERO International Prospective Register of Systematic Reviews (CRD42020172773) (56) (Additional file 2).

\section{Literature search}

The search will follow a comprehensive, sequential three step search strategy, (58)

The first step will be an initial limited search that will include two most relevant online databases (MEDLINE/PubMed and CINAHL), we will identify the key words in the title and abstract of retrieved papers, and the index terms used to describe the articles.

In the second search, we will use all identified keywords and index terms to search across different databases. All search terms used for the search will be added in the appendix. Various boolean operators (AND/OR/NOT), truncations, wildcards etc. either individually or in combination will be used to ensure the comprehensiveness of the search process.

The electronic databasethat will be searched are,

- MEDLINE/PubMed

- ScienceDirect 
- Cochrane database

- Embase

Thirdly, the reference list of identified, relevant articles will be searched for additional studies. In case of missing information/incomplete data, the authors of primary studies will be contacted, if required. A review of Google`

Scholar will also be conducted to include all relevant articles.

The quality of this search strategy will be assessed by at least two independent researchers using the Peer Review of Electronic Search Strategies Evidence-Based Checklist (PRESS EBC) (59). Checklist will be attached in the appendix for reference.

Study designs, particularly, randomized and quasi-randomized controlled trials (RCTs/CCTs), controlled before and after studies (CBAs) and interrupted time series (ITSs), cohort studies will be included. Previous systematic reviews will be analyzed for cross-referencing.

Citation Manager: RefWorks

\section{Study selection}

All titles and abstracts of potentially relevant studies in English, reporting impact/efficacy/effectiveness and/or economic aspect of ASP in a hospital setting in Middle Eastern countries will be vetted by one author (BT). Abstracts will be excluded if they do not fulfil the inclusion criteria. After the screening, all selected research articles will be subjected to full-text review, and independently vetted by 2 reviewers (BT and PAR) and quality assessment will be performed. Any inconsistencies and disagreements will be resolved by consensus or a third reviewer (WK).

\section{Inclusion criteria}

In this review, we have adopted the IDSA definition of ASP (described previously). Studies will be included if any (i) antimicrobial stewardship interventions are (detailed in search terms) applied; (ii) to improve antimicrobial prescribing and/or utilization; and (iii) within an inpatient hospital setting.

In terms of evaluating the economic outcomes of an ASP program, we will use the strategy previously identified by Dik et al (60) that has been widely used in previous literature.

Exclusion criteria includes animal studies or antibiotics used for agricultural needs, data from nonhospital settings such as outpatient/ambulatory care settings or long-term residential care. All non-peer reviewed outputs, narrative reviews, conference proceedings, commentaries, editorials, or qualitative studies will be excluded. Considering the recent developments in terms of implementing stewardship programs as well as within health economics, studies published before 2000 will be excluded.

\section{Data extraction}


Data abstraction will be performed by two independent reviewers (BT \& PAR) using a standardized template which will include three main categories, (i) general characteristics of the reviewed studies such as, country, year of publication, study objective(s), study design, type of hospital, number of beds, number of patients. (ii) Identification of different ASP interventions or tools, based on the categories reported in Dik et al., and finally (iii) all variables linked to the outcome measures listed in the PICO (table 2) will be extracted.

As mentioned earlier, the interventions of an ASP will be classified into three main categories (a) persuasive (b) restrictive and (c) structural, which includes interventions such as, (i) education and training; (ii) pre-authorization, prospective audit, feedback, review, consultation and recommendation; (iii) drug control or monitoring, formulary restriction and intravenous-to-oral conversion; (iv) computerized system use; (v) intervention by infectious disease experts; (vi) antibiotic rotation; (vii) institutional guideline; (viii) national guideline; and (ix) infection control and hand hygiene program.

In terms of outcome measures,

Outcome 1 (Antimicrobials): antimicrobial consumption, before and after the ASP interventions will be recorded, consumptions will be measured in daily dose per 1000 patient days (DDD/1000PD). The efficacy will be determined and expressed in percentage in change of consumptions. Other outcome measures will be de-escalation or shift to targeted therapy, treatment duration, intravenous to oral switch, dose adjustment, and therapeutic drug monitoring (TDM)

Outcome 2 (Clinical): effect of ASP on a series of clinical outcomes includes, measure of resistance, infection related mortality, length of stay, compliance to prescribing of antibiotics with local or national guidelines, incidence of antimicrobial resistance, infection related mortality, compliance to the ASP guidelines, rate of healthcare-associated $\mathrm{C}$. difficile infections, hospital readmission due to relapse of infection within $30 \mathrm{~d}$ after discharge, incidence of multidrug-resistant organisms (MDRO)

Outcome 3 (Economic): the data extraction of economic outcome will include reports on cost of implementation of ASP, operational costs, direct cost related to antimicrobial consumptions, morbidity, and/or mortality costs, length of stay cost, or any overall cost savings reported.

\section{Risk of bias assessment and Quality assessment}

Pairs of reviewers will independently assess quality of each of the studies (BT \& PAR). The Cochrane Risk of Bias tool (61) will be used for randomized controlled trials and for nonrandomized studies we plan to use the Newcastle-Ottawa Quality Assessment Scale (NOS) (62). NOS is a three-dimensional appraisal tool to assess the methodological quality that includes, (i) selected population, (ii) comparability of groups, and (iii) outcome/exposure of interest. The studies will be scored on a scale ranging $0-9$, wherein, a score $>=6$ indicates high-quality studies, a score between 3- 6 as moderate and a score less than 3 will be considered as low quality. In terms of assessing the level of quality of the papers including economic impact, a separate tool, i.e. Consensus on Health Economics Criteria [CHEC] list (12) quality 
criteria will be used. The research team has also proposed the use of ICROMS (Integrated quality Criteria for the Review Of Multiple Study designs), an integrated quality assessment checklist that can be used for a large range of study designs including qualitative research (63).

\section{Data analysis/synthesis}

A narrative synthesis method (64) is deemed most appropriate, where all included studies will be summarized in a narrative form. Tables and graphs will be created to illustrate the key study characteristics such as (population characteristics, study outcomes, sample sizes, settings, methods used, results, and any other important aspect related to each research question of interest). The number of studies and proportions reporting an improvement will be computed. Economic analysis will be conducted based on the following criteria:

Antimicrobial Cost Analysis (ACA) - will include all studies that explicitly reported direct cost of antimicrobial consumption.

Cost-Benefit Analyses (CBA) - will include studies that considered the effects of two different methods and the effects were expressed in terms of cost.

Cost-Effectiveness Analyses (CEA) - will include studies that evaluated relative cost and effects of two different methods.

Cost-Consequence Analyses (CCA) - will include studies that reported different costs and effects of two methods.

Cost-Analyses (CA) - studies that looked at only the cost but not effects of the methods.

Cost-Minimization Analyses (CMA) - studies that looked at cost differences assuming same effects between the two different methods.

If meta-analysis of outcome data is deemed appropriate, random or fixed effect methods will be considered depending on heterogeneity among the studies. Statistical heterogeneity between studies will be assessed using tau squared and $\mathrm{I}^{2}$ statistics and $\mathrm{P}<0.10$ and $\mathrm{I}^{2}>$ than $50 \%$, will be considered as a high level of statistical heterogeneity between the studies. Only, if the studies are homogenous, data will be pooled for a meta-analysis, if otherwise, it will be presented in standardized mean difference (SMD) (65).

\section{Quality of evidence}

Two authors will independently assess the quality of evidence for each outcome. The quality of evidence will be determined by Grading of Recommendations Assessment, Development, and Evaluation (GRADE) system. The studies will be rated based on their evidence ranging high, moderate, low, or very low level. Individual outcomes will be assessed for the following aspects, limitations, inconsistency, indirectness, imprecision, and publication bias. 


\section{Discussion}

Antimicrobial resistance is a global public health concern and healthcare facilities across the world has implemented antimicrobial stewardship as an essential tool to combat resistance. Whilst majority of studies on antimicrobial stewardship has originated from western countries little is known about its clinical and economic impact in the middle east. To the best of our knowledge, this will be the first systematic review to systematically investigate a range of antimicrobial stewardship interventions implemented across hospitals in middle east and to summarise the clinical and economic impact of these interventions on public health. We anticipate that this review will contribute valuable information in terms of antimicrobial stewardship programs to healthcare policymakers, public health practitioners, key stake holders and researchers. Furthermore, the identification of any gaps in the existing literature in terms of combating antimicrobial resistance through antimicrobial stewardship programs will help policy makers, researchers and funding bodies to provide appropriate interventions.

A scoping review has identified that there is considerable degree of heterogeneity between the studies, most notably in terms of study design, exposure measurement, characteristics of participants, and outcomes. Hence it may be difficult to pool data for meta-analysis and thus the data will be tabulated and narrative synthesis will be performed.

To ensure a wider dissemination, the interim findings will be presented at local and international conferences and we aim publish the findings in high impact open access journals. Furthermore, a summary of findings will be disseminated to key stakeholders, policymakers, antimicrobial stewardship committee, hospital infection control committee, etc.

\section{Strengths and limitations of this study}

- To the best of our knowledge, this will be the first review to use a validated systematic and transparent methodological process to appraise and summarize the existing literature on clinical and economic impact of antimicrobial stewardship in the middle east.

- The proposed systematic review is in accordance to the Preferred Reporting Items for Systematic Reviews and Meta Analyses guidelines (PRISMA), ensuring consistency and uniformity in reporting the full systematic review.

- Two independent reviewers will screen the eligible studies and perform extraction and quality assessment to minimize potential reviewer bias.

- One key limitation of this review is it will only include studies published in English, there are chances that we may miss information published in Arabic, Turkish or Persian languages.

- There is a possibility of reporting bias, as we plan to exclude no studies based on the quality, and there is a possibility that findings originate from low and inconsistent studies. However, all the studies will be analyzed for risk of bias and quality of evidence in accordance with GRADE criteria.

\section{List Of Abbreviations}




\begin{tabular}{|ll|}
\hline PRESS EBC & Electronic Search Strategies Evidence-Based Checklist \\
\hline NOS & Newcastle-Ottawa Quality Assessment Scale \\
\hline CDC & Centers for Disease Control and Prevention \\
\hline PROSPERO & The International Prospective Register of Systematic Reviews \\
\hline PRISMA-P & The Preferred Reporting Item for Systematic Review and Meta-analysis \\
\hline GRADE & Grading of Recommendations Assessment, Development, and Evaluation \\
\hline
\end{tabular}

\section{Declarations}

\section{Ethics}

No ethics approval is required for this protocol as it is based on information from the published data.

\section{Consent for publication}

No consent is required as this review is based on information obtained from previously published data.

\section{Acknowledgements}

The study team would like to acknowledge PROSPERO International prospective register of systematic reviews and the editorial for an expedited review.

\section{Author's contribution}

All authors have made substantial contributions to the conception or design, acquisition, analysis, or interpretation of data for the review. MH (Pharmacy Executive Director) has contributed to the conceptualization of this review along with PAR, BT, WK, and DS. BT is clinical pharmacy specialist/doctoral researcher, and coordinator to the antimicrobial stewardship program who took the lead in writing the manuscript. PAR is the head of drug information center and Assistant Director at HMC, will be involved in the literature search and quality assessment. WK is the co-head of the medication safety program and a pharmacy administrative, involved in the acquisition of the financial support, and verification for the validity of content. DS is professor of Pharmacy practice from Qatar University and has vast experience in systematic review and assessing the methodological quality. FH is an obstetrician involved in data collection, application of inclusion/exclusion. HS is an infectious disease consultant and will contribute to synthesis and provide inputs to the antimicrobial findings. RS is an expert medical statistician who will be involved in metaanalysis. ZN and ZAC are assistant professors at Qatar University and will be involved in database and literature search. All authors reviewed and approved the final version of the manuscript prior to submission.

\section{Funding}


The project will seek funding from the Hamad Medical Corporation's Medical Research Center.

\section{Competing interests}

All authors declare no conflict of interest.

\section{References}

1. Tiong JJ, Loo JS, Mai C. Global antimicrobial stewardship: A closer look at the formidable implementation challenges. Frontiers in microbiology. 2016; 7:1860.

2. Prestinaci F, Pezzotti P, Pantosti A. Antimicrobial resistance: a global multifaceted phenomenon. Pathogens and global health. 2015; 109(7):309-318.

3. Lawrence R and Jeyakumar E. Antimicrobial resistance: a cause for global concern. Antimicrobial resistance: a cause for global concern. BMC proceedings: BioMed Central; 2013. p. S1.

4. Vincent J, Rello J, Marshall J, Silva E, Anzueto A, Martin CD, et al. International study of the prevalence and outcomes of infection in intensive care units. Jama. 2009; 302(21):2323-2329.

5. Davey P, Brown E, Charani E, Fenelon L, Gould IM, Holmes A, et al. Interventions to improve antibiotic prescribing practices for hospital inpatients. Cochrane Database of Systematic Reviews. 2013; (4).

6. Chioro A, Coll-Seck AM, Høie B, Moeloek N, Motsoaledi A, Rajatanavin R, et al. Antimicrobial resistance: a priority for global health action. 2015; .

7. Jindal A, Pandya K, Khan I. Antimicrobial resistance: A public health challenge. medical journal armed forces india. 2015; 71(2):178-181.

8. Naylor NR, Atun R, Zhu N, Kulasabanathan K, Silva S, Chatterjee A, et al. Estimating the burden of antimicrobial resistance: a systematic literature review. Antimicrobial Resistance \& Infection Control. 2018; 7(1):58.

9. Ahmad M, Khan AU. Global economic impact of antibiotic resistance: a review. Journal of global antimicrobial resistance. 2019; .

10. Bloom G, Merrett GB, Wilkinson A, Lin V, Paulin S. Antimicrobial resistance and universal health coverage. BMJ global health. 2017; 2(4):e000518-2017-000518. eCollection 2017.

11. Cole J. Antimicrobial resistance-a 'rising tide' of national (and international) risk. The Journal of hospital infection. 2016; 92(1):3-4.

12. Evers $S$, Goossens $M$, De Vet $H$, Van Tulder $M$, Ament A. Criteria list for assessment of methodological quality of economic evaluations: Consensus on Health Economic Criteria. International Journal of Technology Assessment in Health Care. 2005; 21(2):240-245.

13. Karanika S, Paudel S, Grigoras C, Kalbasi A, Mylonakis E. Systematic Review and Meta-analysis of Clinical and Economic Outcomes from the Implementation of Hospital-Based Antimicrobial Stewardship Programs. Antimicrobial Agents and Chemotherapy. 2016; 60(8):4840-4852.

14. McGowan JE,Jr. Economic impact of antimicrobial resistance. Emerging infectious diseases. 2001; 7(2):286-292. 
15. Shrestha P, Cooper BS, Coast J, Oppong R, Thuy NDT, Phodha T, et al. Enumerating the economic cost of antimicrobial resistance per antibiotic consumed to inform the evaluation of interventions affecting their use. Antimicrobial Resistance \& Infection Control. 2018; 7(1):98.

16. Wenzel RP. The antibiotic pipeline-challenges, costs, and values. New England Journal of Medicine. 2004; 351(6):523-526.

17. Holloway KA. Combating inappropriate use of medicines. Expert review of clinical pharmacology. 2011; 4(3):335-348.

18. Schuts EC, Hulscher ME, Mouton JW, Verduin CM, Stuart JWC, Overdiek HW, et al. Current evidence on hospital antimicrobial stewardship objectives: a systematic review and meta-analysis. The Lancet infectious diseases. 2016; 16(7):847-856.

19. Papastergiou V, Georgopoulos SD, Karatapanis S. Treatment of Helicobacter pylori infection: meeting the challenge of antimicrobial resistance. World journal of gastroenterology. 2014; 20(29):98989911.

20. Jacobs MR and Dagan R. Antimicrobial resistance among pediatric respiratory tract infections: clinical challenges. Antimicrobial resistance among pediatric respiratory tract infections: clinical challenges. Seminars in pediatric infectious diseases: Elsevier; 2004. p. 5-20.

21. Sood S, Malhotra M, Das B, Kapil A. Enterococcal infections \& antimicrobial resistance. Indian journal of medical research. 2008; 128(2):111.

22. Hwang AY, Gums JG. The emergence and evolution of antimicrobial resistance: Impact on a global scale. Bioorganic \& medicinal chemistry. 2016; 24(24):6440-6445.

23. Centers for Disease Control and Prevention. Antibiotic resistance threats in the US, 2013. Google Scholar There is no corresponding record for this reference. 2014; .

24. Center for Disease Dynamics, Economics and Policy. State of the World's Antibiotics, 2015. 2015; .

25. Al-Mulla NA, Taj-Aldeen SJ, El Shafie S, Janahi M, Al-Nasser AA, Chandra P. Bacterial bloodstream infections and antimicrobial susceptibility pattern in pediatric hematology/oncology patients after anticancer chemotherapy. Infection and drug resistance. 2014; 7:289-299.

26. Balkhy H, El-Saed A, Al Johani S, Francis C, Al-Qahtani A, Al-Ahdal M, et al. The epidemiology of the first described carbapenem-resistant Klebsiella pneumoniae outbreak in a tertiary care hospital in Saudi Arabia: how far do we go? European journal of clinical microbiology \& infectious diseases. 2012; 31(8):1901-1909.

27. Balkhy HH, El-Saed A, Maghraby R, Al-Dorzi HM, Khan R, Rishu AH, et al. Drug-resistant ventilator associated pneumonia in a tertiary care hospital in Saudi Arabia. Annals of thoracic medicine. 2014; 9(2):104-111.

28. Infectious Diseases Society of America (IDSA). Combating antimicrobial resistance: policy recommendations to save lives. Clinical Infectious Diseases. 2011; 52(suppl_5):S397-S428.

29. McConnell J. European action on antimicrobial resistance. Lancet (London, England). 2001; 358(9295):1787-6736(01)06850-7. 
30. Aslam B, Wang W, Arshad MI, Khurshid M, Muzammil S, Rasool MH, et al. Antibiotic resistance: a rundown of a global crisis. Infection and drug resistance. 2018; 11:1645-1658.

31. Taylor J, Hafner M, Yerushalmi E, Smith R, Bellasio J, Vardavas R, et al. Estimating the economic costs of antimicrobial resistance: model and results. 2014; .

32. Shrivastava SR, Shrivastava PS, Ramasamy J. World health organization releases global priority list of antibiotic-resistant bacteria to guide research, discovery, and development of new antibiotics. Journal of Medical Society. 2018; 32(1):76.

33. Dellit TH, Owens RC, McGowan JE, Gerding DN, Weinstein RA, Burke JP, et al. Infectious Diseases Society of America and the Society for Healthcare Epidemiology of America guidelines for developing an institutional program to enhance antimicrobial stewardship. Clinical Infectious Diseases. 2007; 44(2):159-177.

34. Lee CF, Cowling BJ, Feng S, Aso H, Wu P, Fukuda K, et al. Impact of antibiotic stewardship programmes in Asia: a systematic review and meta-analysis. Journal of Antimicrobial Chemotherapy. 2018; 73(4):844-851.

35. Nasr Z, Paravattil B, Wilby KJ. The impact of antimicrobial stewardship strategies on antibiotic appropriateness and prescribing behaviours in selected countries in the Middle East: a systematic review. Eastern Mediterranean health journal. 2017; 23(6).

36. Pollack LA, Srinivasan A. Core elements of hospital antibiotic stewardship programs from the Centers for Disease Control and Prevention. Clinical Infectious Diseases. 2014; 59(suppl_3):S97S100.

37. Bertollo LG, Lutkemeyer DS, Levin AS. Are antimicrobial stewardship programs effective strategies for preventing antibiotic resistance? A systematic review. American Journal of Infection Control. 2018; 46(7):824-836.

38. Drekonja DM, Filice GA, Greer N, Olson A, MacDonald R, Rutks I, et al. Antimicrobial stewardship in outpatient settings: a systematic review. infection control \& hospital epidemiology. 2015; 36(2):142152.

39. Kaki R, Elligsen M, Walker S, Simor A, Palmay L, Daneman N. Impact of antimicrobial stewardship in critical care: a systematic review. Journal of antimicrobial chemotherapy. 2011; 66(6):1223-1230.

40. Wagner B, Filice GA, Drekonja D, Greer N, MacDonald R, Rutks I, et al. Antimicrobial stewardship programs in inpatient hospital settings: a systematic review. Infection Control \& Hospital Epidemiology. 2014; 35(10):1209-1228.

41. Dyar O, Pagani L, Pulcini C. Strategies and challenges of antimicrobial stewardship in long-term care facilities. Clinical Microbiology and Infection. 2015; 21(1):10-19.

42. Alghamdi S, Shebl NA, Aslanpour Z, Shibl A, Berrou I. Hospital adoption of antimicrobial stewardship programmes in Gulf Cooperation Council countries: a review of existing evidence. Journal of global antimicrobial resistance. 2018; 15:196-209.

43. Thomas B, Paudyal V, MacLure K, Pallivalapila A, McLay J, El Kassem W, et al. Medication errors in hospitals in the Middle East: a systematic review of prevalence, nature, severity and contributory 
factors. European journal of clinical pharmacology. 2019; 75(9):1269-1282.

44. Khalifeh MM, Moore ND, Salameh PR. Self-medication misuse in the Middle East: a systematic literature review. Pharmacology research \& perspectives. 2017; 5(4):e00323.

45. Alhomoud F, Aljamea Z, Almahasnah R, Alkhalifah K, Basalelah L, Alhomoud FK. Self-medication and self-prescription with antibiotics in the Middle East-do they really happen? A systematic review of the prevalence, possible reasons, and outcomes. International journal of infectious diseases. 2017; 57:3-12.

46. Sakeena M, Bennett AA, McLachlan AJ. Non-prescription sales of antimicrobial agents at community pharmacies in developing countries: a systematic review. International journal of antimicrobial agents. 2018; 52(6):771-782.

47. Dameh M, Green J, Norris P. Over-the-counter sales of antibiotics from community pharmacies in Abu Dhabi. Pharmacy world \& science. 2010; 32(5):643-650.

48. Almohammed RA, Bird EL. Public knowledge and behaviours relating to antibiotic use in Gulf Cooperation Council countries: A systematic review. Journal of infection and public health. 2019; 12(2):159-166.

49. Jose J, Jimmy B, Alsabahi AG, Al Sabei GA. A study assessing public knowledge, belief and behavior of antibiotic use in an omani population. Oman medical journal. 2013; 28(5):324-330.

50. Dandachi I, Chaddad A, Hanna J, Matta J, Daoud Z. Understanding the Epidemiology of Multi-Drug Resistant Gram-Negative Bacilli in the Middle East Using a One Health Approach. Frontiers in microbiology. 2019; 10:1941.

51. Habibzadeh F. Use and misuse of antibiotics in the Middle East. Lancet. 2013; 382(1).

52. Abdelrahman T, Alsaeed M, Karam R, Alkhthami A, Alswat O, Alzahrani A, et al. Misuse of antibiotics and antibiotic resistance: a public population-based health survey in Altaif-Saudi Arabia. WJPMR. $2017 ; 2: 54-62$.

53. Awad Al, Aboud EA. Knowledge, attitude and practice towards antibiotic use among the public in Kuwait. PloS one. 2015; 10(2):e0117910.

54. Belkina T, Al Warafi A, Eltom EH, Tadjieva N, Kubena A, Vlcek J. Antibiotic use and knowledge in the community of Yemen, Saudi Arabia, and Uzbekistan. The Journal of Infection in Developing Countries. 2014; 8(04):424-429.

55. Moienzadeh A, Massoud T, Black E. Evaluation of the general public's knowledge, views and practices relating to appropriate antibiotic use in Q atar. International Journal of Pharmacy Practice. 2017; 25(2):133-139.

56. Hashad N, Tonna A, Perumal D, Stewart D. Antimicrobial stewardship program implementation in the middle east: a systematic review protocol. [homepage on the Internet]. PROSPERO International prospective register of systematic reviews; 2017 [updated December 19; cited 2020 March/26]. Available from: https://www.crd.york.ac.uk/prospero/display_record.php?RecordID=79597.

57. Moher D, Shamseer L, Clarke M, Ghersi D, Liberati A, Petticrew M, et al. Preferred reporting items for systematic review and meta-analysis protocols (PRISMA-P) 2015 statement. Systematic reviews. 
2015; 4(1):1.

58. Aromataris E, Riitano D. Constructing a search strategy and searching for evidence. A guide to the literature search for a systematic review. The American Journal of Nursing. 2014; 114(5):49-56.

59. McGowan J, Sampson M, Salzwedel DM, Cogo E, Foerster V, Lefebvre C. PRESS peer review of electronic search strategies: 2015 guideline statement. Journal of clinical epidemiology. 2016; 75:4046.

60. Dik JH, Vemer P, Friedrich AW, Hendrix R, Lo-Ten-Foe JR, Sinha B, et al. Financial evaluations of antibiotic stewardship programs-a systematic review. Frontiers in microbiology. 2015; 6:317.

61. Higgins JP, Altman DG, Gotzsche PC, Juni P, Moher D, Oxman AD, et al. The Cochrane Collaboration's tool for assessing risk of bias in randomised trials. BMJ (Clinical research ed.). 2011; 343:d5928.

62. Wells G, Shea B, O'connell D, Peterson J, Welch V, Losos M, et al. The Newcastle-Ottawa Quality Assessment Scale (NOS) for assessing the quality of nonrandomized studies in meta-analyses. Clin Epidemiol [Internet]. 2017; :1-2.

63. Zingg W, Castro-Sanchez E, Secci F, Edwards R, Drumright L, Sevdalis N, et al. Innovative tools for quality assessment: integrated quality criteria for review of multiple study designs (ICROMS). Public health. 2016; 133:19-37.

64. Schünemann HJ, Oxman AD, Higgins JP, Vist GE, Glasziou P, Guyatt GH. Presenting results and 'Summary of findings' tables. Cochrane handbook for systematic reviews of interventions. 2008; 5:0.

65. Deeks JJ, Higgins JP, Altman DG, Cochrane Statistical Methods Group. Analysing data and undertaking meta-analyses. Cochrane handbook for systematic reviews of interventions. 2019; :241284.

\section{Tables}

Table 1: Core Elements of Hospital Antibiotic Stewardship Programs 


\begin{tabular}{|ll|}
\hline Core Elements & Requirements \\
Leadership & Dedicating necessary human, financial, and information technology resources \\
\hline Accountability & $\begin{array}{l}\text { Appointing a single leader responsible for program outcomes and accountable to } \\
\text { an executive-level or patient quality-focused hospital committee. Experience with } \\
\text { successful programs shows that a physician or pharmacist leader is effective }\end{array}$ \\
\hline Drug expertise & $\begin{array}{l}\text { Appointing a single pharmacist leader responsible for working to improve antibiotic } \\
\text { use }\end{array}$ \\
\hline Action & $\begin{array}{l}\text { Implementing at least 1 recommended action, such as systemic evaluation of } \\
\text { ongoing treatment needs after a set period of initial treatment (ie, antibiotic "time- } \\
\text { out" after 48 h }\end{array}$ \\
\hline Tracking & $\begin{array}{l}\text { Monitoring process measures (eg, adherence to facility-specific guidelines, time to } \\
\text { initiation or de-escalation), impact on patients (eg, Clostridium difficile infections, } \\
\text { antibiotic-related adverse effects and toxicity), antibiotic use and resistance }\end{array}$ \\
\hline Education & $\begin{array}{l}\text { Regular reporting of the above information to doctors, nurses, and relevant staff } \\
\text { Educating clinicians about disease state management, resistance, and optimal } \\
\text { prescribing }\end{array}$ \\
\hline
\end{tabular}

Source: Centers for Disease Control and Prevention

Table 2: Framework for determining eligibility of research questions 


\title{
Criteria Determinants
}

Population Patients receiving antimicrobials in hospitals

$(\mathrm{P})$

Interventions

(I)
Antimicrobial stewardship interventions the interventions of an ASP will be classified into three main categories (a) persuasive (b) restrictive and (c) structural, which includes interventions such as, (i) education and training; (ii) pre-authorization, prospective audit, feedback, review, consultation and recommendation; (iii) drug control or monitoring, formulary restriction and intravenous-to-oral conversion; (iv) computerized system use; (v) intervention by infectious disease experts; (vi) antibiotic rotation; (vii) institutional guideline; (viii) national guideline; and (ix) infection control and hand hygiene program.

Comparison

(C)

No comparison as such, however few studies might have included before and after analysis, or hospitals with vs without ASPs

\section{Outcome (0) Antimicrobial}

Antimicrobial consumption (DDD/1000 patient days)

De-escalation or shift to targeted therapy

Treatment duration

Antimicrobial choice

Intravenous to oral switch

Dose adjustment

Therapeutic drug monitoring (TDM)

\section{Clinical}

Length of stay

Antimicrobial resistance

Infection related mortality

Compliance to the ASP guidelines

Rate of healthcare-associated C. difficile infections

Hospital readmission due to relapse of infection within $30 \mathrm{~d}$ after discharge

Incidence of multidrug-resistant organisms (MDRO)

\section{Economic}

\author{
Implementation cost \\ Operational cost \\ Antimicrobial expenditure
}


- Morbidity, and/or mortality costs (costs associated with hospital procedures, treatment etc.)

- Length of stay cost

- Overall cost saving

Setting (S) Data from hospital inpatients

\section{Supplementary Files}

This is a list of supplementary files associated with this preprint. Click to download.

- Internationalprospectiveregisterofsystematicreviews.pdf

- PRISMAchecklist.doc 